\title{
(GIGA)bYte
}

\section{Ubiquitin-proteasome pathway annotation in Diaphorina citri can reveal potential targets for RNAi-based pest management}

\author{
Will Tank ${ }^{1}$, Teresa Shippy ${ }^{1}$, Amanda Thate ${ }^{1}$, Crissy Massimino ${ }^{2}$, \\ Prashant S. Hosmani ${ }^{3}$, Mirella Flores-Gonzalez ${ }^{3}$, Lukas A. Mueller ${ }^{3}$, \\ Wayne B. Hunter ${ }^{4}$, Susan J. Brown ${ }^{1}$, Tom D’Elia ${ }^{2}$ and Surya Saha ${ }^{3,5, *}$ \\ 1 Division of Biology, Kansas State University, Manhattan, KS 66506, USA \\ 2 Indian River State College, Fort Pierce, FL 34981, USA \\ 3 Boyce Thompson Institute, Ithaca, NY 14853, USA \\ 4 USDA-ARS, US Horticultural Research Laboratory, Fort Pierce, FL 34945, USA \\ 5 Animal and Comparative Biomedical Sciences, University of Arizona, Tucson, AZ 85721, USA
}

\section{ABSTRACT}

Ubiquitination is an ATP-dependent process that targets proteins for degradation by the proteasome. Here, we annotated 15 genes from the ubiquitin-proteasome pathway in the Asian citrus psyllid, Diaphorina citri. This psyllid vector has come to prominence in the last decade owing to its role in the transmission of the devastating bacterial pathogen, Candidatus Liberibacter asiaticus (CLas). Infection of citrus crops by this pathogen causes Huanglongbing (HLB), or citrus greening disease, and results in the eventual death of citrus trees. The identification and correct annotation of these genes in $D$. citri will be useful for functional genomic studies to aid the development of RNAi-based management strategies aimed at reducing the spread of HLB. Investigating the effects of $C$ Las infection on the expression of ubiquitin-proteasome pathway genes may provide new information about the role these genes play in the acquisition and transmission of $C$ Las by $D$. citri.

Submitted: Accepted: Published:

12 October 2021 26 February 2022 02 March 2022

* Corresponding author. E-mail: suryasaha@cornell.edu

Published by GigaScience Press. Preprint submitted at https: //doi.org/10.1101/2021.10.11.464014

Included in the series: Asian citrus psyllid community annotation (https://doi.org/10.46471/GIGABYTE_ SERIES_0001)

This is an Open Access article distributed under the terms of the Creative Commons Attribution License (https://creativecommons. org/licenses/by/4.0/), which permits unrestricted reuse, distribution, and reproduction in any medium, provided the original work is properly cited.

Gigabyte, 2022, 1-10
Subjects Genetics and Genomics, Animal Genetics, Bioinformatics

\section{DATA DESCRIPTION}

The ubiquitin-proteasome pathway (Figure 1) is responsible for the targeted degradation of most proteins in eukaryotic cells [1]. It regulates the concentration of proteins and degrades misfolded proteins. Ubiquitin modification is an ATP-dependent process. Ubiquitination is initiated by ubiquitin-activating enzymes (E1), which activate and transfer ubiquitin to ubiquitin-conjugating enzymes (E2).

Conjugation of proteins with ubiquitin is mediated by ubiquitin ligases (E3) and tags the target protein for degradation by the proteasome. In its active form, the complete $26 \mathrm{~S}$ proteasome comprises a $20 \mathrm{~S}$ catalytic cylindrical core, and one or two $19 \mathrm{~S}$ regulatory subunits bound to the termini on either or both sides of the core (Figure 2) [3].

The 195 regulatory subunits, which are made up of Rpn and Rpt subunits, bind ubiquitinated proteins and may help to unfold the proteins while translocating them to the $20 \mathrm{~S}$ catalytic core. The $20 \mathrm{~S}$ core, often referred to as the $20 \mathrm{~S}$ proteasome, comprises two $\beta$-rings and two $a$-rings that organize into a cylinder-shaped quaternary structure. 


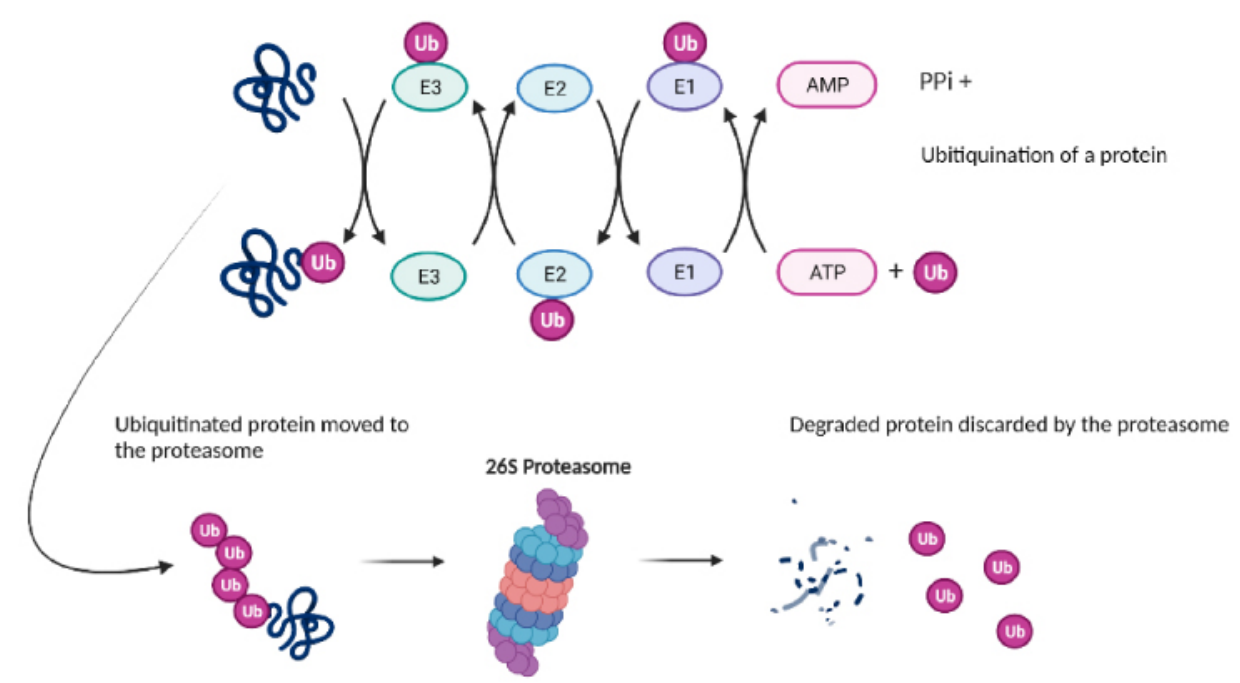

Figure 1. Ubiquitination process and interaction with the proteasome. Created with BioRender [2]. Ub: Ubiquitin.

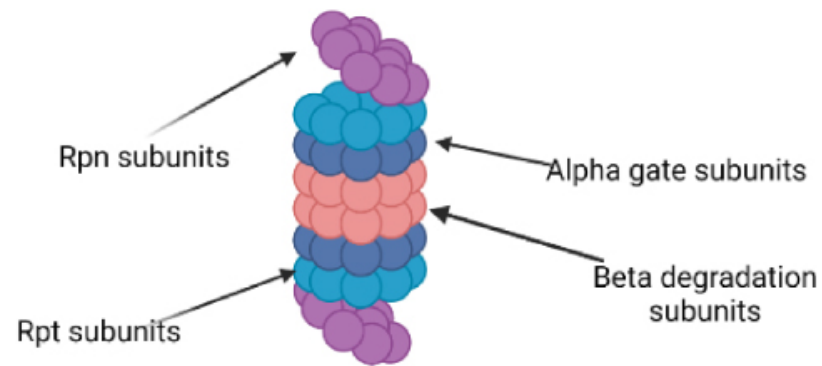

Figure 2. Proteasome and its subunits. Created with BioRender [2].

Proteolysis occurs at the N-termini of the $\beta$-rings where threonine residues form active sites that hydrolyze proteins via threonine nucleophilic hydrolysis [3].

\section{CONTEXT}

The ubiquitin-proteasome pathway is highly conserved among eukaryotes and has been the target of many investigations attempting to decode the functions and inner workings of this pathway [1]. Studies involving arthropods [4] have linked the ubiquitin-proteasome pathway to development, immune response, gametogenesis, differentiation, apoptosis and stress response [5]. For example, the main pathways involved in insect resistance to viral and bacterial infections, Toll and JAK/STAT, are both dependent on proteasome activity for degradation of ubiquitinated targets [6, 7].

In this study, we identified and manually annotated 15 genes from the ubiquitin-proteasome pathway in Diaphorina citri (NCBI:txid121845), which is the vector for Candidatus Liberibacter asiaticus (CLas), a bacterial pathogen associated with citrus greening disease. Several studies have suggested that the ubiquitin-proteasome pathway is affected by $C$ Las infection of $D$. citri. Ramsey et al. [8] found that several ubiquitin-proteasome pathway proteins are differentially expressed between $C$ Las( $\left.{ }^{+}\right)$and 


\begin{tabular}{|c|c|c|c|c|c|}
\hline \multirow[b]{2}{*}{ Gene } & \multirow[b]{2}{*}{ OGS 3.0 identifier } & \multicolumn{4}{|c|}{ Evidence supporting annotation } \\
\hline & & MCOT & Iso-seq & RNA-seq & Ortholog \\
\hline Ubiquitin Thioesterase (OTU1) & Dcitr07g12810.1.1 & & $\mathrm{X}$ & & $\mathrm{X}$ \\
\hline Ubiquitin conjugating enzyme 7 (Ubc7) & Dcitr05g04710.1.1 & & $\mathrm{X}$ & & $\mathrm{X}$ \\
\hline Regulatory particle non-ATPase (Rpn3) & Dcitr04g11565.1.1 & & $\mathrm{x}$ & & $\mathrm{x}$ \\
\hline Small ubiquitin-related modifier (smt3) & Dcitr02g04830.1.1 & MCOT07264.0.TT & $\mathrm{X}$ & & $\mathrm{X}$ \\
\hline Ubiquitin-specific protease 7 (Usp7) & Dcitr07g06000.1.1 & & $\mathrm{x}$ & & $\mathrm{X}$ \\
\hline Ubiquitin-like activating enzyme 5 (Uba5) & Dcitr01g11870.1.1 & $\begin{array}{l}\text { MCOT04043.0.CT } \\
\text { MCOT19464.0.CT } \\
\text { MCOT01465.4.CT }\end{array}$ & & $\mathrm{x}$ & $\mathrm{X}$ \\
\hline E3 ubiquitin-protein ligase (Bre1) & Dcitr08g07680.1.1 & МСОТ14294.0.СТ & & $\mathrm{x}$ & $\mathrm{X}$ \\
\hline Regulatory particle triple-A ATPase 3 (Rpt3) & Dcitr06g06690.1.1 & МСОТ00775.0.СТ & & $\mathrm{x}$ & $\mathrm{x}$ \\
\hline Regulatory particle non-ATPase 7 (Rpn7) & Dcitr06g05390.1.1 & MCOT18826.2.CT & & & $\mathrm{X}$ \\
\hline Regulatory particle non-ATPase 11 (Rpn11) & Dcitr01g16470.1.1 & MCOT21540.0.СT & & & $\mathrm{X}$ \\
\hline Regulatory particle non-ATPase 12 (Rpn12) & Dcitr09g09680.1.1 & МСОТ03904.0.СТ & $\mathrm{X}$ & $\mathrm{x}$ & $\mathrm{X}$ \\
\hline Proteasome a6 subunit (Prosa6) & Dcitr06g06380.1.1 & & & & $\mathrm{X}$ \\
\hline Ubiquitin activating enzyme 1 (Uba1) & Dcitr03g10190.1.1 & МСОТ05061.0.СТ & & & $\mathrm{x}$ \\
\hline Proteasome $\beta 6$ subunit (Pros $\beta 6)$ & Dcitr05g14710.1.1 & MCOT17488.0.CO & & & $\mathrm{X}$ \\
\hline Proteasome $\beta 5$ subunit (Pros $\beta 5)$ & Dcitr06g03790.1.1 & & $\mathrm{X}$ & & $\mathrm{X}$ \\
\hline
\end{tabular}

CLas(-) D. citri, suggesting that protein degradation may be affected by CLas infection. Two other studies [9] reported differential expression of multiple E3 ubiquitin ligases during CLas infection [10]. With the purpose of finding possible pest control targets, Ulrich et al. [11] noted that several ubiquitin-proteasome genes were among the 40 genes associated with the highest lethality percentages in an RNA interference (RNAi) screen of the beetle Tribolium castaneum [12], suggesting that this pathway is a possible target for RNAi-based control of insects.

\section{METHODS}

Ubiquitin-proteasome genes in D. citri genome v3.0 [13] were identified through BLAST (NCBI BLAST, RRID:SCR_004870) searches of $D$. citri sequences with ubiquitin-proteasome orthologs primarily from Drosophila melanogaster. Orthology was then verified by reciprocal BLAST of the National Center for Biotechnology Information (NCBI) non-redundant protein database [14]. Genes were manually annotated and validated in Apollo 2.1.0 (RRID:SCR_001936) using available evidence, including RNA-seq reads, Iso-seq and MCOT (MAKER (RRID:SCR_005309), Cufflinks (RRID:SCR_014597), Oases (RRID:SCR_011896), and Trinity (RRID:SCR_013048)) [13] (Table 1). A neighbor-joining phylogenetic tree was constructed in MEGA version 10 (Mega, RRID:SCR_000667) using full-length protein sequences from D. citri, T. castaneum [12] and D. melanogaster [7] (Table 2), employing the CLUSTALW (ClustalW, RRID:SCR_017277) multiple sequence alignment with Poisson correction method and 1000 replicate bootstrap test.

Table 3 shows a list of orthologs evaluated for gene validation. Expression data from the Citrus Greening Expression Network (CGEN) were visualized using the pheatmap [16] (pheatmap, RRID:SCR_016418) package of R (R Project for Statistical Computing, RRID:SCR_001905) or Microsoft Excel (Microsoft Excel, RRID:SCR_016137).

Further details on the annotation process can be found at protocols.io [15] (Figure 3). 


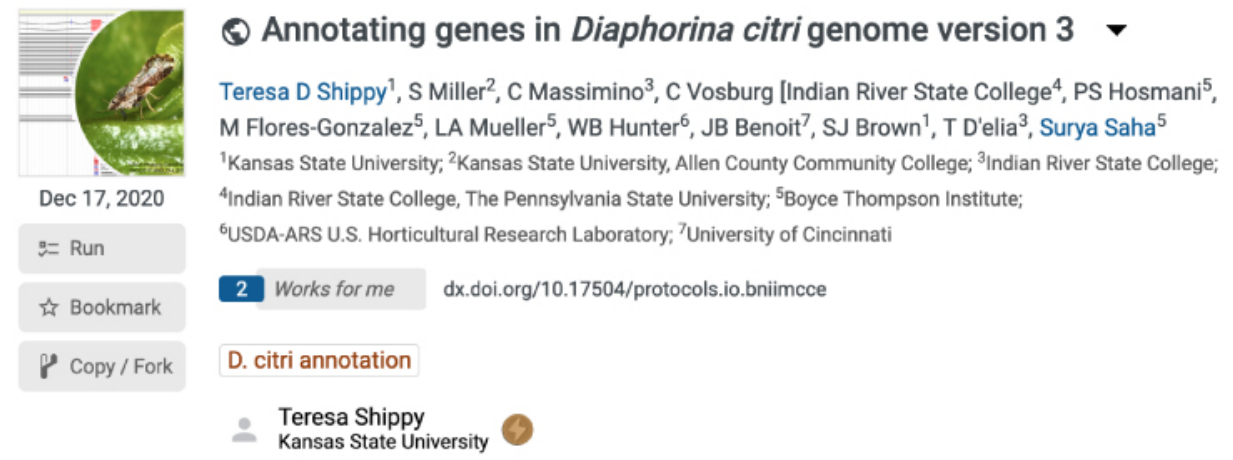

Figure 3. Protocols.io protocol for annotating genes in Diaphorina citri genome version 3 [15]. https://www. protocols.io/widgets/doi?uri=dx.doi.org/10.17504/protocols.io.bniimcce

Table 2. NCBI accession numbers for ortholog proteasome subunit annotations.

\begin{tabular}{lccc}
\hline Gene & Diaphorina citri & Tribolium castaneum & Drosophila melanogaster \\
Rpt3 & Dcitr06g06690.1.1 & XP_967976.1 & NP_001285108.1 \\
Rpn3 & Dcitr04g11565.1.1 & XP_971686.1 & NP_001260557.1 \\
Rpn7 & Dcitr06g05390.1.1 & XP_973643.1 & XP_033162910.1 \\
Rpn11 & Dcitr01g16470.1.1 & XP_008196636.1 & NP_608905.1 \\
Rpn12 & Dcitr09g09680.1.1 & XP_971959.1 & NP_648904.1 \\
Prosa6 & Dcitr06g06380.1.1 & XP_966973.1 & NP_001285798.1 \\
Pros 66 & Dcitr05g14710.1.1 & XP_968855.1 & NP_524115.1 \\
Pros $\beta 5$ & Dcitr06g03790.1.1 & XP_970194.1 & NP_652014.1 \\
\hline
\end{tabular}

Table 3. Count of orthologous genes in representative insects.

\begin{tabular}{|c|c|c|c|c|}
\hline Gene & $\begin{array}{c}\text { Drosophila } \\
\text { melanogaster }\end{array}$ & $\begin{array}{c}\text { Tribolium } \\
\text { castaneum }\end{array}$ & $\begin{array}{c}\text { Acyrthosiphon } \\
\text { pisum }\end{array}$ & Diaphorina citri \\
\hline OTU1 & 1 & 1 & 1 & 1 \\
\hline Ubc7 & 1 & 1 & 1 & 1 \\
\hline Rpn3 & 1 & 1 & 1 & 1 \\
\hline Smt3 & 1 & 1 & 1 & 1 \\
\hline Usp7 & 1 & 1 & 1 & 1 \\
\hline Uba5 & 1 & 1 & 1 & 1 \\
\hline Bre1 & 1 & 1 & 1 & 1 \\
\hline Rpt3 & 1 & 1 & 1 & 1 \\
\hline Rpn7 & 1 & 1 & 1 & 1 \\
\hline Rpn 11 & 1 & 1 & 1 & 1 \\
\hline Rpn 12 & 1 & 1 & 1 & 1 \\
\hline Prosa6 & 1 & 1 & 1 & 1 \\
\hline Uba1 & 1 & 1 & 1 & 1 \\
\hline Pros $\beta 6$ & 1 & 1 & 1 & 1 \\
\hline Pros $\beta 5$ & 1 & 1 & 1 & 1 \\
\hline
\end{tabular}

\section{DATA VALIDATION AND QUALITY CONTROL}

The ubiquitin-proteasome pathway contains hundreds of genes. However, in our study we focused on the genes identified by Ramsey et al. [8] and Ulrich et al. [11]. We identified a 
single ortholog of each of the ubiquitin-proteasome pathway genes described in those studies (Tables 2 and 3), which is consistent with the gene count in D. melanogaster [7], T. castaneum [12] and Acyrthosiphon pisum. Genes were named based on their Drosophila orthologs. Since Ramsey et al. [8] observed differences in protein levels between CLas(+) and CLas(-) insects for the products of several of these genes, we used CGEN [18] to compare transcriptional expression data from publicly available RNA-seq samples of CLas(+) and CLas(-) D. citri.

Two main groups of genes were annotated in this study: ubiquitination-related genes, and proteasome-related genes encoding proteasome-subunits.

\section{UBIQUITINATION-RELATED GENES}

\section{Ubiquitin activating enzyme 1 (Uba1)}

As the only E1 enzyme in the ubiquitination pathway of insects, Uba1 is critical for initiation of the ubiquitination process [19]. In D. melanogaster, clones of cells homozygous for mutations in Uba1 show changes in the regulation of apoptosis, with weak alleles causing reduced apoptosis and strong alleles causing increased apoptosis [19]. Cells adjacent to clones for strong alleles of Uba1 overproliferate because of increased Notch signaling, suggesting that Uba1, through its role in ubiquitination, may act as a tumor suppressor gene. Flies completely lacking Uba1 do not survive. However, flies homozygous for weaker alleles sometimes live to adulthood, but have reduced lifespan and motor defects [20]. Knockdown of Uba1 in Tribolium also causes high levels of lethality [11], suggesting that Uba1 could be a good target for RNAi-based pest control.

\section{Ubiquitin conjugating enzyme 7 (Ubc7)}

Ubc7 is one of about $30 \mathrm{E} 2$ ubiquitin-conjugating enzymes known in D. melanogaster. It is also known as courtless, becauses male flies lacking Ubc7 display abnormal courtship behavior, as well as defective spermatogenesis [21]. In D. citri, Ramsey et al. [8] observed almost six-fold higher expression of Ubc7 in CLas(+) organisms (Figure 4). To determine if this difference could be caused by changes in transcription, we analyzed $U b c 7$ expression in publicly available RNA-seq data in CGEN. Comparison of $U b c 7$ transcript levels in CLas(+) versus $C$ Las(-) samples did show slight upregulation of $U b c 7$ in some CLas (+) samples.

\section{E3 ubiquitin-protein ligase (Bre1)}

As one of the E3 ligase genes, Bre1 is a highly conserved gene affecting several other pathways through its role in the ubiquitin pathway. For example, in D. melanogaster, the Notch signaling pathway requires Bre1 for histone modification [22]. In CLas(+) psyllids, Bre1 protein levels were greatly reduced (almost 50-fold) compared with CLas(-) psyllids. However, no significant change in transcript levels is seen in the available RNA-seq data (Figure 4).

\section{Ubiquitin Thioesterase (OTU1)}

In D. melanogaster, Ubiquitin Thioesterase 1 (OTU1) is a hydrolase responsible for removal of conjugated ubiquitin and regulates protein turnover by halting the ubiquitination process and retaining proteins [23]. In D. citri, Ramsey et al. [8] observed differential expression of OTU1 during CLas infection, with $C$ Las $(+)$ psyllids showing 13-fold higher expression than $C$ Las(-). In nymphs fed on CLas(+) Citrus spp., we saw an approximately 


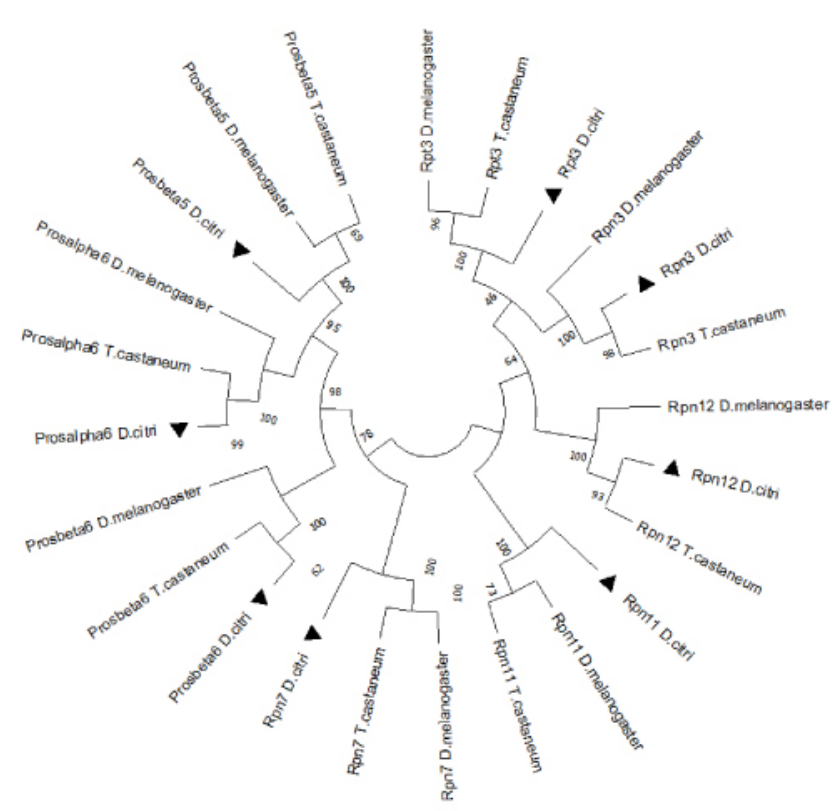

Figure 4. Phylogenetic comparison of proteasome subunit orthologs. The numbers are bootstrap values and the D. citri proteins are marked with a triangle.

six-fold increase in OTU1 transcripts compared with nymphs fed on uninfected plants of the same species. However, this was a single replicate study and none of the other CLas(+) versus CLas(-) comparisons showed a significant difference in OTU1 expression (Figure 4).

\section{Ubiquitin-specific protease 7 (Usp7)}

Usp7 is another deubiquitinase/protease, and has been demonstrated in D. melanogaster to regulate aging and autophagy [24]. In D. melanogaster, specimens with knocked down Usp7 have difficulty coping with oxidative stress and starvation [24]. Furthermore, Usp7 has been shown to interact with the p53 tumor suppressor gene [25] and inhibition of Usp7 through small molecule targeted approaches have shown promise in increasing cytotoxicity and inducing tumor cell death [25]. In contrast to OTU1, which also negatively regulates ubiquitin-mediated degradation, Usp7 protein showed three-fold downregulation in CLas(+) organisms [8]. We saw no evidence of differential expression at the transcript level (Figure 4).

\section{Ubiquitin-like activating enzyme 5 (Uba5)}

Uba5 is an E1 enzyme for the ufmylation pathway, which modifies proteins in a manner similar to the ubiquitination pathway [26]. In ufmylation, the ubiquitin-like protein Ubiquitin fold modifier 1 (UFM1) is conjugated to target proteins. The functions of ufmylation are not well understood, but it seems to be important for tumor suppression, response to DNA damage, and regulation of protein homeostasis in the endoplasmic reticulum [27]. Absence of Uba5 in D. melanogaster causes cerebellar ataxia, resulting in severe locomotive defects and a shortened lifespan [28]. Uba5 shows an almost three-fold reduction in protein levels in CLas(+) than CLas(-) psyllids [8]. However, there seems to be no consistent effect of CLas infection on Uba5 transcript levels (Figure 4). 


\section{Small ubiquitin-related modifier (smt3)}

The smt3 gene encodes the Small Ubiquitin-Like Modifier (SUMO) protein [28]. Conjugation of SUMO to target proteins, known as sumoylation, occurs by a process analogous to ubiquitination, but uses a distinct set of E1, E2 and E3 enzymes. Sumoylation has the opposite effect of ubiquitination in that it inhibits protein degradation [29, 30]. Studies in D. melanogaster have shown that sumoylation may potentiate the immune response against infections [29]. Ramsey et al. [8] observed a four-fold reduction in D. citri Smt3 protein in CLas(+) versus CLas(-) psyllids. We do see a reduction in smt3 transcript levels in adult psyllids fed on Citrus spp. infected with CLas, but this difference is not seen in nymphs or in adults fed on Citrus medica (Figure 4).

\section{PROTEASOME GENES}

\section{Regulatory particle non-ATPase (Rpn3), Regulatory particle non-ATPase 7 (Rpn7), Regulatory particle non-ATPase 11 (Rpn11), Regulatory particle non-ATPase 12 (Rpn12)}

The Regulatory particle non-ATPase (Rpn) subunits of the proteasome are responsible for the 'lid' complex of the proteasome. They are directly responsible for capturing polyubiquitinated proteins and moving them into the cylindrical complex [3]. Furthermore, Rpn11 has been recognized as being responsible for deubiquitinating these proteins. RNAi knockdown of Rpn subunits in Nilaparvata lugens, a member of the same insect order as D. citri, caused multiple defects in female reproduction, suggesting that the proteolytic activity of the proteasome is important for these processes [31]. Moreover, Rpn7, Rpn11 and Rpn12 were shown by both Ulrich et al. [11] and Knorr et al. [32] to be potential high lethality RNAi targets in T. castaneum.

\section{Regulatory particle triple-A ATPase 3 (Rpt3)}

The six Regulatory particle triple-A ATPase (Rpt) subunits are organized into a hexameric ring, responsible for unfolding the proteins and opening the a gates within the proteasome, where the protein will be ultimately degraded [3]. Rpt3 is one of the three subunits that contains the conserved C-terminal hydrophobic-tyrosine-X (HbYX) [3], which has been shown to be directly responsible for facilitating gate opening. In fission yeast, Rpt3 has been associated with CENP-A, a variant of histone $\mathrm{H} 3$ responsible for kinetochore establishment [33, 34]. In RNAi knockdown experiments in Tribolium, Rpt3 was one of the top 11 genes with respect to lethality percentage.

\section{Proteasome a6 subunit (Prosa6), Proteasome $\beta 6$ subunit (Pros $\beta 6$ ), Proteasome $\beta 5$ subunit (Pros $\beta 5$ )}

The Proteasome (Pros) alpha proteins are the 'gating' subunits, which receive the unfolded proteins provided by the Rpt subunits [3]. The Pros beta subunits provide the peptidases and catalytic enzymatic activity that degrade the proteins within the proteasome. In D. melanogaster, mutations in the Pros units cause accentuated muscle degradation and a 25\% decrease in lifespan [35]. The three Pros subunit genes annotated here were identified as good targets for RNAi-based pest control because of high lethality percentages [11].

The protein sequences of the various proteasome subunits are often quite similar, making it challenging to establish orthology. We performed phylogenetic analysis to ensure 
that we had correctly identified all the annotated $D$. citri proteasome subunit genes (Figure 4). All D. citri subunit proteins clustered with the expected D. melanogaster and $T$. castaneum orthologs, indicating that the annotated genes are named correctly.

In Ramsey et al. [8], a very distinct difference can be observed between upregulated and downregulated proteins, with as much as 50 -fold differences in expression. When these data are displayed as a clustered heatmap scaled by gene (Figure 4) we can compare the relative expression levels between samples. There is very little differential expression of genes between $C \operatorname{Las}(+)$ and $C \operatorname{Las}(-)$ samples. The apparent differences between $C$ Las effect on protein and gene expression could indicate that there is post-transcriptional regulation of these genes/proteins. Post-transcriptional regulation of proteasome genes in yeast [25] has been observed before, and could be a possible explanation for these discrepancies.

\section{Conclusion and future work}

The ubiquitin-proteasome pathway is highly conserved in most eukaryotes [1] and, therefore, a potential candidate for comprehensive studies. Disruption of this pathway has shown promise as a pest control mechanism in various insects, as explored by Ramsey et al. [8] and Ulrich et al. [11], among many others. This has led us to annotate 15 ubiquitin-proteasome pathway genes from $D$. citri. Previous reports also suggested that the pathway might be affected by CLas infection [8, 9]. Our analyses found little effect of CLas infection on transcription of the subset of ubiquitin-proteasome genes we annotated. More comprehensive studies will be needed to understand how CLas influences this pathway in D. citri.

\section{RE-USE POTENTIAL}

The models annotated in this work will be helpful to researchers studying this pathway in many organisms, as this pathway is present in most eukaryotic species. Future studies are required to confirm the validity of the ubiquitin-proteasome pathway as an RNAi target for control of $D$. citri populations.

\section{DATA AVAILABILITY}

The $D$. citri genome assembly, official gene sets, and transcriptome data can be downloaded from the Citrus Greening website [36]. Additional datasets supporting this article are available in the GigaScience GigaDB repository [37].

\section{EDITOR'S NOTE}

This article is one of a series of Data Releases crediting the outputs of a student-focused and community-driven manual annotation project curating gene models and if required, correcting assembly anomalies, for the $D$. citri genome project [38].

\section{DECLARATIONS}

\section{LIST OF ABBREVIATIONS}

CLas: Candidatus Liberibacter asiaticus; NCBI: National Center for Biotechnology Information, CGEN: Citrus Greening Expression Network; RNAi: RNA interference; RNA-seq: RNA sequencing; Iso-seq: Isoform sequencing; MCOT: Maker, Cufflinks, Oases, Trinity.

\section{ETHICAL APPROVAL}

Not applicable. 


\section{CONSENT FOR PUBLICATION}

Not applicable.

\section{COMPETING INTERESTS}

The authors declare that they have no competing interests.

\section{FUNDING}

This work was supported by USDA-NIFA grants 2015-70016-23028, HSI 2020-38422-32252 and 2020-70029-33199.

\section{AUTHOR CONTRIBUTIONS}

WBH, SJB, TD and LAM conceptualized the study; TD, SS, TDS and SJB supervised the study; SJB, TD, SS and LAM contributed to project administration; WT, AT and CM conducted the investigation; PH, MF-G and SS contributed to software development; PH, MF-G, SS, TDS and JB developed methodology; SJB, TD, WBH and LAM acquired funding; WT prepared and wrote the original draft; TD, SJB, SS, TDS, CM, WBH and JB reviewed and edited the draft.

\section{REFERENCES}

1 Ferrandon D. Ubiquitin-proteasome: pallbearer carries the deceased to the grave. Immunity, 2007; 541-544. doi:10.1016/j.immuni.2007.10.003.

2 BioRender. 2022; https://biorender.com/. Accessed 10 October 2021.

3 Tanaka K. The proteasome: overview of structure and functions. Proc. Jpn. Acad. Ser. B: Phys. Biol. Sci., 2009; 85(1): 12-36. doi:10.2183/pjab.85.12.

4 Choy A, Severo MS, Sun R et al. Decoding the ubiquitin-mediated pathway of arthropod disease vectors. PLoS One, 2013; 8: 1-12. doi:10.1371/journal.pone.0078077.

5 Mykles DL. Structure and functions of arthropod proteasomes. Mol. Biol. Rep., 1999; 26: 103-111.

6 Severo MS, Sakhon OS, Choy A et al. The "ubiquitous" reality of vector immunology. Cell Microbiol., 2013; 15: 1070-1078.

7 Thurmond J, Goodman JL, Strelets VB et al. FlyBase 2.0: the next generation. Nucleic Acids Res., 2019; 47(D1): D759-D765. doi:10.1093/nar/gky1003.

8 Ramsey JS, Johnson RS, Hoki JS et al. Metabolic interplay between the Asian Citrus Psyllid and its profftella symbiont: an Achilles' heel of the citrus greening insect vector. PLoS One, 2015; 10: e0140826. doi:10.1371/journal.pone.0140826.

9 Jaiswal D, Sidharthan VK, Sharma SK et al. Candidatus Liberibacter asiaticus manipulates the expression of vitellogenin, cytoskeleton, and endocytotic pathway-related genes to become circulative in its vector, Diaphorina citri (Hemiptera: Psyllidae). 3 Biotech., 2021; 11(2): 88. doi:10.1007/s13205-021-02641-x.

10 Yu HZ, Li NY, Zeng XD et al. Transcriptome analyses of Diaphorina citri midgut responses to Candidatus liberibacter asiaticus infection. Insects, 2020; 11: 171.

11 Ulrich J, Dao VA, Majumdar U et al. Large scale RNAi screen in Tribolium reveals novel target genes for pest control and the proteasome as prime target. BMC Genom., 2015; 16: 1-9. doi:10.1186/s12864-015-1880-y.

12 Richards S, Gibbs RA, Weinstock GM et al. Tribolium Genome Sequencing Consortium. The genome of the model beetle and pest Tribolium castaneum. Nature, 2008; 452: 949-955.

13 Hosmani PS, Flores-Gonzalez M, Shippy T et al. Chromosomal length reference assembly for Diaphorina citri using single-molecule sequencing and Hi-C proximity ligation with manually curated genes in developmental, structural and immune pathways. bioRxiv. 2019; https://doi.org/10.1101/869685. Cold Spring Harb. Lab.

14 National Center for Biotechnology Information (NCBI). Protein database. 2019; https://www.ncbi.nlm.nih.gov/protein.

15 Shippy T, Miller S, Massimino C et al. Annotating genes in Diaphorina citri genome 3. protocols.io. 2020; https://dx.doi.org/10.17504/protocols.io.bniimcce. 
16 Kolde R. Pretty Heatmaps. 2020; https://cran.r-project.org/package=pheatmap. Accessed 07 January 2021.

17 FlyBase. https://flybase.org/. Accessed 01 October 2021.

18 Boyce Thompson Institute. Citrus Greening Expression Network. https://cgen.citrusgreening.org.

19 Lee TV, Ding T, Chen Z et al. The E1 ubiquitin-activating enzyme Uba1 in Drosophila controls apoptosis autonomously and tissue growth non-autonomously. Development, 2008; 135(1): 43-52. doi:10.1242/dev.011288

20 Liu H-Y, Pfleger CM. Mutation in E1, the ubiquitin activating enzyme, reduces Drosophila lifespan and results in motor impairment. PLoS One, 2013; 8(1): e32835. doi:10.1371/journal.pone.0032835.

21 Orgad S, Rosenfeld G, Greenspan RJ et al. courtless, the Drosophila UBC7 homolog, is involved in male courtship behavior and spermatogenesis. Genetics, 2000; 155(3): 1267-1280. doi:10.1093/genetics/155.3.1267.

22 Bray S, Musisi H, Bienz M. Bre1 is required for notch signaling and histone modification. Dev. Cell, 2005; 8(2): 279-286. doi:10.1016/j.devcel.2004.11.020.

23 Messick TE, Russell NS, Iwata AJ et al. Structural basis for ubiquitin recognition by the Otu1 ovarian tumor domain protein. J. Biol. Chem., 2008; 283(16): 11038-11049. doi:10.1074/jbc.M704398200.

24 Cui L, Song W, Zeng Y et al. Deubiquitinase USP7 regulates Drosophila aging through ubiquitination and autophagy. Aging (Albany NY), 2020; 12(22): 23082-23095. doi:10.18632/aging.104067.

25 Kategaya L, Di Lello P, Rougé L et al. USP7 small-molecule inhibitors interfere with ubiquitin binding. Nature, 2017; 550: 534-538. doi:10.1038/nature24006.

26 Komatsu M, Chiba T, Tatsumi K et al. A novel protein-conjugating system for Ufm1, a ubiquitin-fold modifier. EMBO J., 2004; 23(9): 1977-1986. doi:10.1038/sj.emboj.7600205.

27 Banerjee S, Kumar M, Wiener R. Decrypting UFMylation: how proteins are modified with UFM1. Biomolecules, 2020; 10(10): 1442. doi:10.3390/biom10101442.

28 Duan R, Shi Y, Yu L et al. UBA5 mutations cause a new form of autosomal recessive cerebellar ataxia. PLoS One, 2016; 11(2): e0149039. doi:10.1371/journal.pone.0149039.

29 Bhaskar V, Smith M, Courey AJ. Conjugation of Smt3 to dorsal may potentiate the Drosophila immune response. Mol. Cell. Biol., 2002; 22(2): 492-504. doi:10.1128/MCB.22.2.492-504.2002.

30 Handu M, Kaduskar B, Ravindranathan R et al. SUMO-enriched proteome for drosophila innate immune response. G3 (Bethesda), 2015; 5(10): 2137-2154. doi:10.1534/g3.115.020958.

31 Wang W, Yang RR, Peng LY et al. Proteolytic activity of the proteasome is required for female insect reproduction. Open Biol., 2021; 11(2): 200251. doi:10.1098/rsob.200251.

32 Knorr E, Fishilevich E, Tenbusch $L$ et al. Gene silencing in Tribolium castaneum as a tool for the targeted identification of candidate RNAi targets in crop pests. Sci. Rep., 2018; 8: 1-15. doi:10.1038/s41598-018-20416-y.

33 Kitagawa T, Ishii K, Takeda K et al. The 19S proteasome subunit Rpt3 regulates distribution of CENP-A by associating with centromeric chromatin. Nat. Commun., 2014; 5: 3597. doi:10.1038/ncomms4597.

34 Cheeseman IM. The kinetochore. Cold Spring Harb. Perspect. Biol., 2014; 6(7): a015826. doi:10.1101/cshperspect.a015826.

35 Langerak S, Kim M, Lamberg H et al. The Drosophila TGF-beta/activin-like ligands Dawdle and Myoglianin appear to modulate adult lifespan through regulation of $26 \mathrm{~S}$ proteasome function in adult muscle. Biol. Open., 2018; 7(4): bio029454. doi:10.1242/bio.029454.

36 Citrus Greening Solutions. 2018; https://citrusgreening.org/.

37 Tank W, Shippy TD, Thate A et al. Supporting data for "Ubiquitin proteasome pathway annotation in Diaphorina citri can reveal potential targets for RNAi based pest management”. GigaScience Database. 2022; http://dx.doi.org/10.5524/100995.

38 Asian citrus psyllid community annotation series. GigaByte. 2022; https://doi.org/10.46471/GIGABYTE_SERIES_0001. 